\title{
Fixation Artefact and Intensity of Histological Staining: Observations on Mammalian Liver Cells ${ }^{1}$
}

\author{
Atuhiro Sibatani and Hiroto Naora \\ Microbial Diseases Research Institute, University of Osaka, and \\ Department of Physics, Faculty of Science, University of Tokyo
}

Received September 15, 1953

The appearance of dark cells and light cells in fixed sections of mammalian liver had long been a subject of much discussion among morphologists until Scharrer (1938) proved that it is entirely due to an artefact. He found that the complete perfusion of the liver with fixing solutions such as formalin, Bouin's fluid, or Zenker-formalin does not cause the dark and light cells to appear, while tissue pieces cut from the liver and then immersed in these fixatives frequently show groups of such cells, especially along the cut surface and around the blood vessels of the liver. This finding led him to assume that the dark cells which were shrunken and the light cells which were swollen were both artifical products, the deformation being caused mechanically by pressing and pulling when the test pieces were being cut from the liver tissue.

In our morphologic studies on mammalian livers, we also experienced cases in which the appearance of dark and light cells could be explained most satisfactorily if we assumed that they were artefacts. But our results indicated that the artefact of this type was due to fixation with formalin or formalin-containing fixatives rather than to mechanical cutting of the liver. Since the problem of dark cells has a certain"connexion to some topics dealt with in recent literature on liver cytology and nucleoprotein cytochemistry, a brief note on the results of our observations may be pertinent.

As Scharrer has already pointed out, dark and light cells appear more frequently in cases of various liver injuries than in intact livers. But they can consistently be demonstrated in sections prepared from liver slices which have been immersed in distilled water for 0.5 to several hours at room temperature before fixation with formalin. Experiments of this type were performed by Opie (1948) in his systematic studies on the osmotic enlargement of mitochondria of liver cells. He recognized strongly basophilic cells appearing along the cut surface of the liver slices immersed in distilled water; they did not appear in outermost layers of cells but in a deeper zone next to them. Cells in the top zone of these liver slices were non-basophilic and

1 Aided in part by the Scientific Research Fund of the Ministry of Education given to the Synthetic Research Group on "Nucleic Acids".

2 Present address: Department of Cytochemistry and Histology, Yamaguchi Medical School, Nakaube, Ube, Yamaguchi-ken, Japan. 
swollen, while basophilic cells in the deeper zone were rather shrunken. In Opie's plate 7, fig. 7, the shrunken cells are immediately next to the swollen ones, appearing to have been compressed by the latter.

Similar pictures were obtained in our experiments in which slices of adult rat liver were immersed in distilled water for $0.5-3$ hours at room temperature, fixed in 20 per cent tormalin, and stained with Congo rubin, an acidic azo-dye, without any counterstain. Alteration of cell structure is found along the intact free surface, and to a greater extent, along the cut surface of the liver. In accordance with Opie's observations, several zones
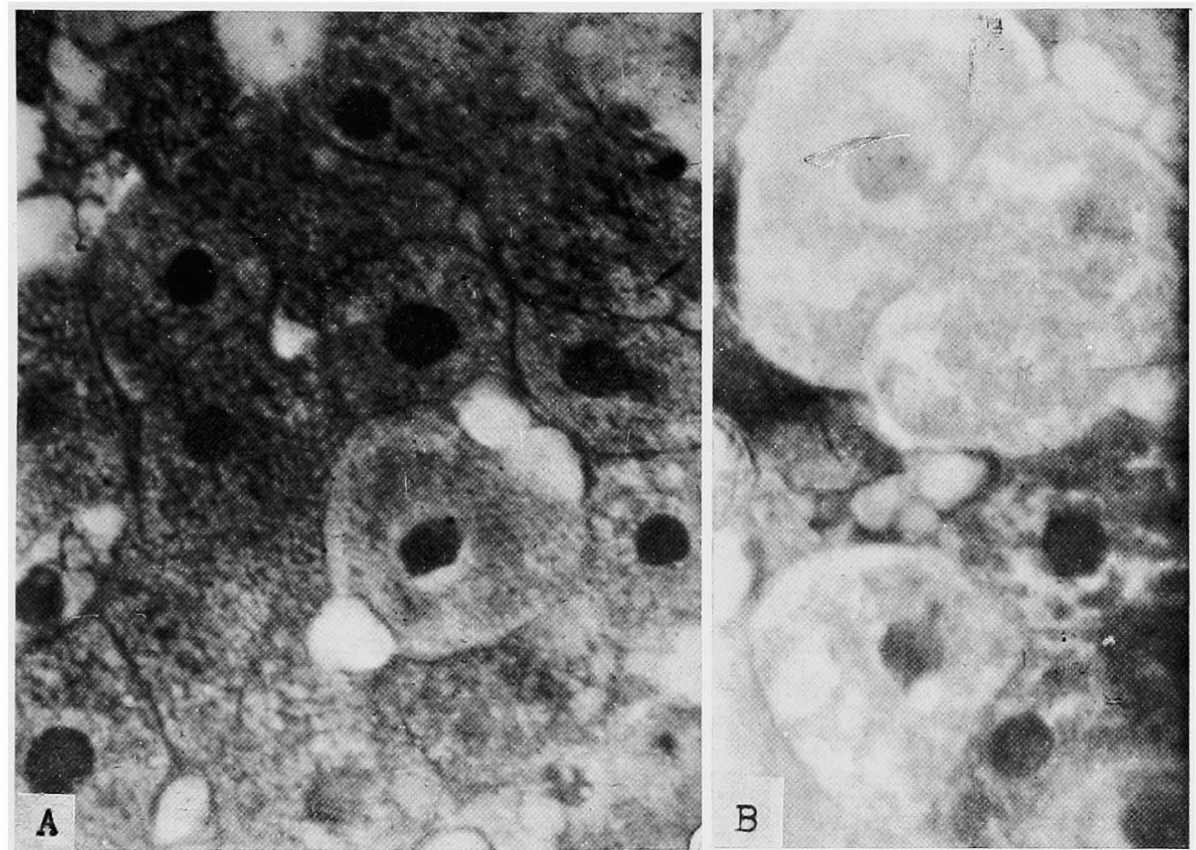

Fig. 1. Cells of a rat liver slice fixed with formalin after immersing in distilled water for 30 minutes. Congo rubin staining. $\times 770 .-$ A. Foam-like cells with mitochondria swollen and vesicular. From the external zone of the cut surface. Note the heavy staining of nuclei. In the centre there is a slightly swollen cell which stains a little weaker than the other cells. In this cell appears the change leading to the light cell artefact to have taken place to a certain extent.-B. Three light cells (2 above and 1 below) are extremely swollen. In between densely stained, shrunken, dark cells are seen. Mitochondria are not swollen in either type of cells. From the top layers of free margin of the liver.

are to be distinguished at the cut surface. A few layers of cells in the outermost zone are partially disintegrated; in lower level there are 1-8 layers of cells with osmotically swollen mitochondria which give the cytoplasm a foam-like appearance (these cells will be called below foam-like cells, Fig. 1A). The cells of this type become more shrunken inwardly, with gradually finer meshes of swollen mitochondria. Next to this zone, further in, there appear extremely shrunken cells which seem to be compressed by neibouring cells, and heavily stainable (dark cells, Fig. 1B). Mitochondria of dark cells may 
not be swollen. Intermingled with them markedly enlarged cells with rarefied cytoplasm are seen, and it is these cells that seem to be compressing the dark cells by their enlarged cell bodies (light cells, Fig. 1B). Dark and light cells may occur in a roughly alternate sequence, forming 1-2 layers of cells, which are bordered inward with the main field of the liver tissue involving almost intact cells (Fig. 2A). Along the free margin of the liver, similar changes are seen, but the altered layers are less thick and partially disintegrated cells found in the cut surface are lacking. With short period of immersion ( 30 minutes) in distilled water, there were only 2 layers of dark and light cells, but on immersion for 3 hours, it was found that a single layer of dark and light cells was bordered outwardly with layers of foam-like cells. It should be pointed out that staining with basic dyes gave essentially the same picture as the one obtained with acidic dyes.

That the formation of foam-like cells is actually due to the action of distilled water was proven by experiments with fresh liver homogenate. Exposure to distilled water of free liver cells obtained by homogenizing the liver with sucrose solution or saline immediately resulted in their conversion into foam-like cells with swollen, vesicular mitochondria. It should thus be concluded that formalin fixation well retains the foam-like cells. The dark and light cells, on the other hand, could not be produced by distilled water in fresh liver homogenate.

It was then found that different fixatives result in the development of dark and light cells to varying degrees in distilled water-immersed liver slices. Among several kinds of fixatives tested, only those containing formalin (1020 per cent formalin, alchol-formalin, Bouin's, Helly's and Regaud's fluids) were found to give rise to the appearance of dark and light cells, while sections fixed with those not containing formalin such as Carnoy's fluid or absolute alcohol showed very few or entirely none of such cells. With freezingdrying technique (Nakazima, Tsujii and Naora, 1950), liver cells at the cut surface were evenly swollen, but there were no definable dark and light cells; the swollen cells apparently represented the foam-like cells, but they did not show any definable foam-like structure of the cytoplasm, probably owing to the ice-crystal formation because of the high water content of these cells (Fig. 2B).

These observations clearly indicate that the formation of dark and light cells is brought about by fixation artefact and not by the action of distilled water itself. The fact that these cells were not found at all in control slices not receiving the treatment with distilled water suggests that this kind of artefacts is due to not only fixation with formalin but also a certain kind of injuries of hepatic cells, which by itself does not cause the appearance of dark and light cells. This idea differs definitely from the Scharrer's view ascribing the artefact to the mechanical action of cutting tissue pieces before fixation. But the fact that the number and, to a certain extent, also the 
distribution of the dark and light cells can be changed artificially after liver pieces have been cut from the tissue would be a sufficient evidence to exclude the Scharrer's postulate.

The mechanism of the formation of these artefacts is not clear; also the question as to whether these artefacts are caused by formalin per se or rather by subsequent procedures such as dehydration with alcohol and so on, in addition to the formalin fixation, remains to be answered. Since light and dark cells usually appear together in group and intermingled, the possibility suggests itself that the swelling of light cells and the shrinkage of dark cells attached to them are not independent, but interrelated phenomena. Also
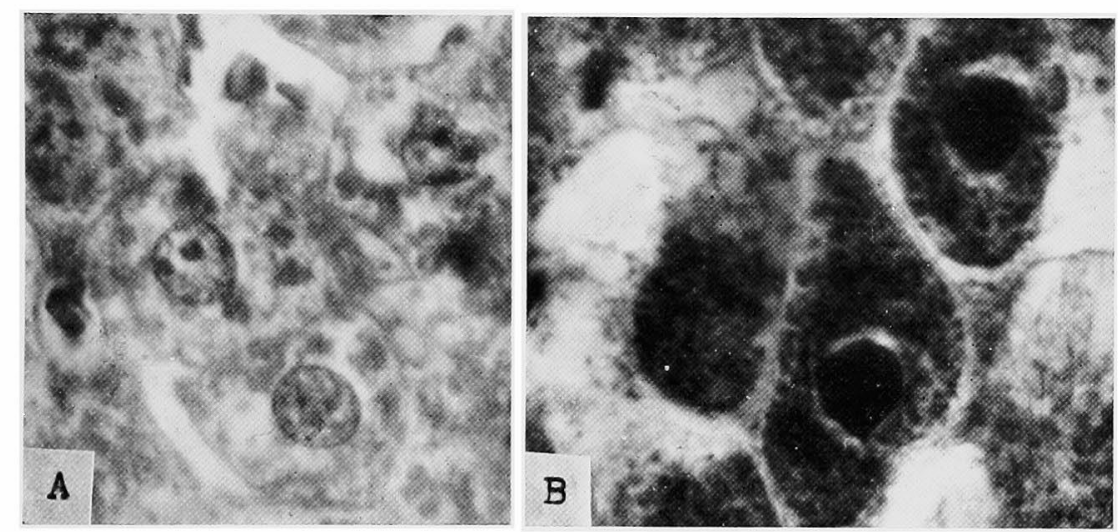

Fig. 2. A. The same as Fig. 1. Almost normal appearance of hepatic cells from the inner field of the liver slice.-B. Cells from the external zone of a liver slice immersed in distilled water for 30 minutes and frozen-dried, maybe representing foam-like cells.

Note the intense staining with Congo rubin of both nuclei and cyto-plasm. $\times 760$.

among foam-like cells, an artefact of this kind seems to occur eventually to a very slight extent (Fig. 1A). From the levels of dark and light cell zone and of foam-like cell zone in liver slices treated with distilled water, it seems that dark and light cells are formed from cells less injured than the external foam-like cells.

These findings are in good agreement with the data of Opie and Scharrer. All the fixatives used by these authors contained formalin. Figures shown by them are quite alike to the pictures we obtained. The fact that the perfusion with fixatives of the liver prevents these artefacts from occurring (Scharrer, 1938) is readily explained by the even and direct action of formalin; it should be recalled that the dark and light cells appear in deeper zones at the cut surface, and not in the outermost cell layers.

We will now turn to the cytochemical aspects of the dark and light cell artefacts. As illustrated by Scharrer, staining with iron hematoxylin is a very sharp test to demonstrate this kind of artefacts. But acidic and basic dyes can as well be utilized to detect it. However, there may be some uncertainty as to whether dark cells demonstrated by iron hematoxylin are quite 
the same as the ones shown by ordinary acidic and basic dyes. If we restrict our discussion to the pictures obtained with acidic and basic dyes, the strong stainability of dark cells could entirely be ascribed to high concentrations of stainable materials (nucleic acids and proteins) in these cells which are markedly shrunken. Likewise the low stainability of light cells seems to be due to its enormous size. Therefore, high and low staining intensities of dark cells and light cells respectively do not imply the accumulation in, or loss from, those cells of the stainable materials. Indeed, such events are most improbable to occur because these cells simply represent the fixation artefacts.

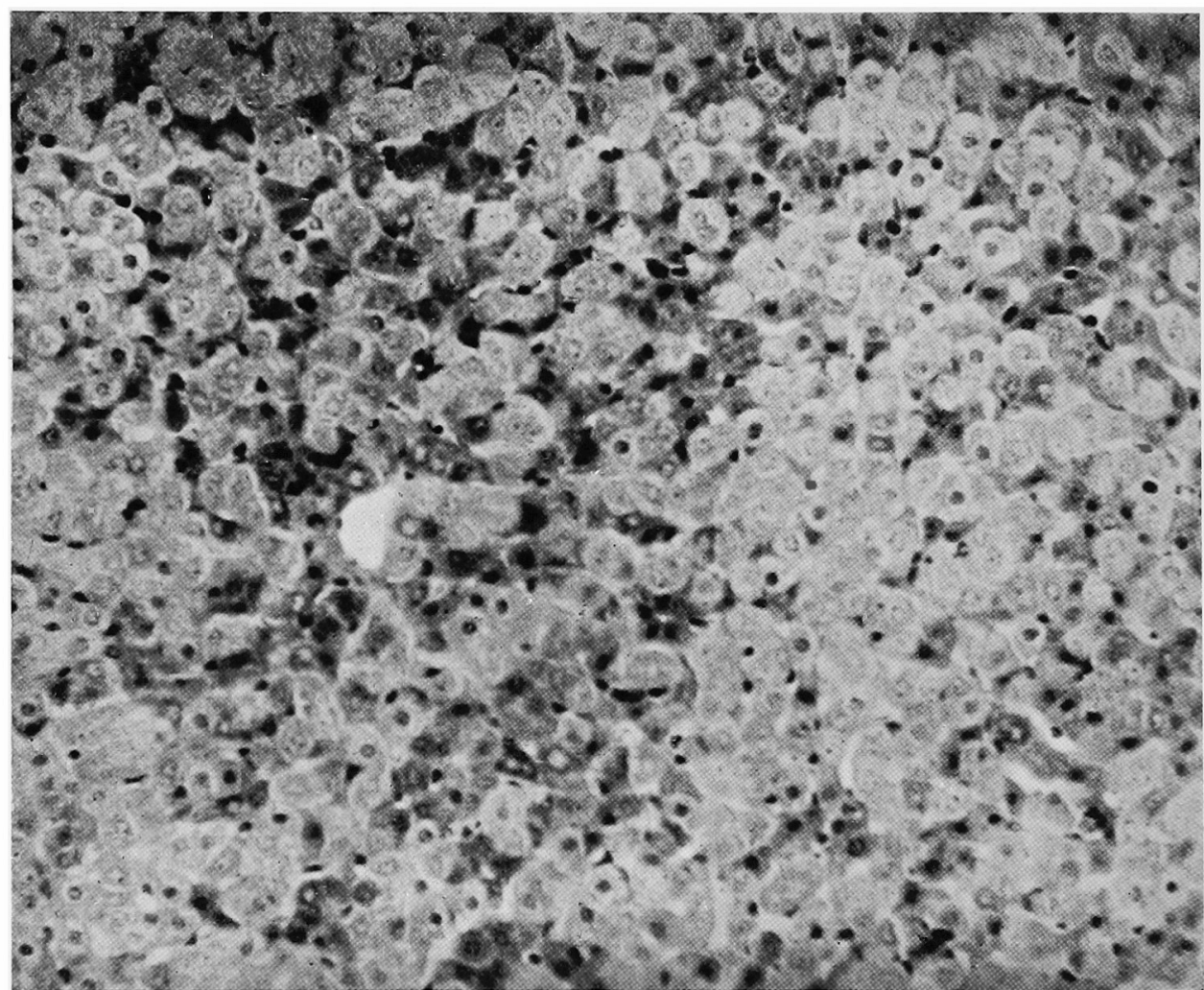

Fig. 3. Section of a guinea pig liver. Thionin staining. $\times 160$. The field containing large numbers of dark and light cells. Note the varying degrees of basophilia of nuclei in different hepatic cells.

Thus, the mass appearance of "basophilic" liver cells as illustrated in Fig. 3 does not mean anything but that the change in cell volume without any alteration in quantity of the stainable materials of cells is to cause more remarkable change in the stainability of cells than might first appear; this is simply because the stainability of cells depends upon the concentration of cell components (mass/volume), and hence inversely proportional to the third power of the cell diameter.

It is clear that the single use of a dye, be it acidic or basic, can more 
faithfully reflect the change in concentration of stainable materials of cells than the combined use of acidic and basic dyes, for electrostatic combination between acidic cell components and basic dyes can well be interfered by the introduction of acidic dyes as competitors into the staining fluid and vice versa. Further we must take into account the masking of potentially stainable materials by oppositely charged cell constituents which are associated with the ones to be stained. Injuries of cells such as immersion in distilled water or fixation with formalin as employed here may well affect such a "natural" association between cell components, and this can be assessed as one of the factors controlling the stainability of cells (cf. Kaufmann, 1951; Mirsky and Ris, 1951; Mazia et al., 1953).

Examples of what was just stated are seen in our illustrations. In Figs. $1 \mathrm{~A}, \mathrm{~B}$ and $2 \mathrm{~B}$, nuclei of most hepatic cells, which must have been severely affected by distilled water, are densely and homogeneously stained with Congo rubin, an acidic dye, while hepatic nuclei of almost intact cells given in Fig. 2A are stained lightly with the same dye, showing definite chromatinic and nucleolar structures. Since accumulation of proteins in nuclei of distilled water-damaged cells is not to be expected and also there is no significant diminution in nuclear volume of these cells, the strong stainability of these nuclei could not be explained in any way but by assuming that the inhibition of staining reaction of proteins through nucleic acids was being reversed here by some mechanism.

A similar situation is found in Fig. 3, which shows the section of a normal guinea pig liver fixed with 50 per cent formalin. Here the staining with thionin, a basic dye, revealed that some of the nuclei of swollen (light) cells and most of the nuclei of dark cells are heavily stainable, while the other nuclei of swollen cells as well as seemingly intact cells stain lightly.

These considerations, then, seem to invalidate the Opie's interpreation with respect to the nature of strong basophilia of dark cells in distilled watertreated liver. Opie assumed that the pentose nucleic acid (PNA) of cells in outermost layers of the slice was extracted by distilled water, migrated inward along with penetrating distilled water and deposited on the cells in lower level. But the shrunken appearance of this "basophilic cells" indicates their identity with our dark cells. And the use by Opie of a modification of Giemsa stain suggests the occurrence of complicate competition between basic cell components and the basic dyes on the one hand and between acidic cell components and the acidic dye on the other hand, in the simultaneous staining reactions with the combined acidic and basic dyes of affected tissues, in which the potentially stainable groups occuluded in unaffected cells may well have been unmasked. In other words, "basophilic" cells of Opie do not imply the increase of PNA per cell, but only suggests the increase in concentration, and, possibly, also the release from nucleoprotein complex, of PNA. 


\section{Summary}

It was demonstrated that the dark cells and light cells encountered in fixed sections of some normal and injured livers can artificially be produced by immersing liver slices in distilled water. The formation of these cells is not brought about by the action of distilled water per se but rather represents an artefact caused by fixation with formalin-containing fixatives, being readily realized at a certain level of cell injuries. The strong stainability of the dark cell and the weak one of the light cell seem to be due to the change in volume rather than in quantity of stainable materials of cells. A possibility of changes in the intensity of nuclear and cytoplasmic staining caused by the unmasking of stainable groups was suggested.

\section{References}

Kaufmann, B. P., MeDonald, M. R. and Gay, H. 1951. J. Cell Comp. Physiol. 38: Suppl. 1, 71 .

Mazia, D., Brewer, P. A. and Alfert, M. 1953. Biol. Bull. 104: 57.

Mirsky, A. E., and Ris, H. 1951. J. Gen. Physiol. 34: 475.

Nakazima, M., Tsujii, and Naora, H. 1950. Science (Japan) 20: 204.

Opie, E. L. 1948. J, Expt. Med. 87: 425.

Scharrer, E. 1938. Anat. Rec. $72: 53$. 